\title{
Mobile Technology \& Nursing Education, Practice
}

\author{
Dr. Hanan Elzeblawy Hassan
}

Lecturer of Maternal \& Newborn Health nursing, Faculty of Nursing, Beni-suef University.

Dr. Shaimaa Hassan Mohamady

Lecturer of Maternal and Newborn Health, Nursing, Faculty of Nursing, Helwan University.

\section{Dr. Hend Abdallah El sayed Afifi}

Lecturer of Obstetrics and Woman Health Nursing, Faculty of Nursing, Benha University 


\section{Presentation talk}

الاستشهاد المرجعي:

Hassan , Hanan Elzeblawy (2016) Mobile Technology \& Nursing Education, Practice/ Hanan Elzeblawy Hassan, Shaimaa Hassan Mohamady \& Hend Abdallah El sayed Afifi.- Journal of Distance Learning and Open Learning .- Vol.4, Issue 6 (Jan. - May).-PP.5-13 


\section{BACKGROUND:}

- Technology is at every corner of every profession. If the nurses are not learning to adapt to the influences of technology, they can miss out on a lot of inventive and innovative techniques for success. This is true for nursing education as well. Many universities and training facilities are implementing the use of technology in nursing education to improve the quality of health care for patients.

- Mobile phones are becoming increasingly important in everyday life and now in healthcare. There has been a steady growth of information and communication technologies in health communication and technology is used progressively in telemedicine, wireless monitoring of health outcomes in disease and in the delivery of health interventions. Mobile phones are becoming an important method of encouraging better nurse-patient communication and will undoubtedly increase in application over coming years.

\section{BENEFITS OF PRESENTATION:}

- This presentation will provide an overview of mobile technology, identify the advantage of mobile, describe the uses of mobile in education and practice in nursing. 


\section{MOBILE ADVANTAGES}

- While the advantage of instant, current information available via mobile devices is fairly obvious, unique features make them an even greater educational tool. Features such as the ability to simultaneously search all software and link from one software reference to another enhance the ability of students to connect, analyze and synthesize disease and patient information.

- The search function provides research capability across references, while linkages connect one source to another.

- Mobile technology allows student nurses to prepare rapidly for patient care at the beginning of each shift within the clinical setting. Nursing faculty report a significant increase in student knowledge and preparation prior to care when they utilize point-of-care resources.

- By utilizing mobile resources for nursing education in the classroom, lab and clinical venues, students develop enhanced skills in critical thinking and clinical decision making.

- Mobile technology for nurses has changed the face of nursing education and clinical practice.

- Simultaneous searching allows nursing students to type in a term on their mobile device and obtain a list of locations in which that term appears.

- Linking features connect related information , among the references.

- Many mobile resources are based on evidence-based practice findings, include illustrations for the multimodal learner and are updated more frequently than their printed counterparts. 
- Due to the depth and breadth of information readily available on mobile devices, students can be more actively engaged in learning in the classroom and clinical laboratory setting through case study work, concept and conceptual care map development and clinical pathway or care plan work that in the past was limited by lack of textbook availability

\section{USES OF MOBILE TECHNOLOGY IN NURSING}

Now this technology is poised to alter how health care is delivered, the quality of the patient experience, and the cost of health care.

- Mobile technology is helping with chronic disease management,

- Empowering the elderly and expectant mothers,

- patient safety

- Administration of medication

- Care planning

- Reminding people to take medication at the proper time, extending service to underserved areas,

- Improving health outcomes and medical system efficiency.

- Curriculum integration

- Patient education 

shnology\& ...

Managing Chronic Diseases through Remote Monitoring and Mobile Devices

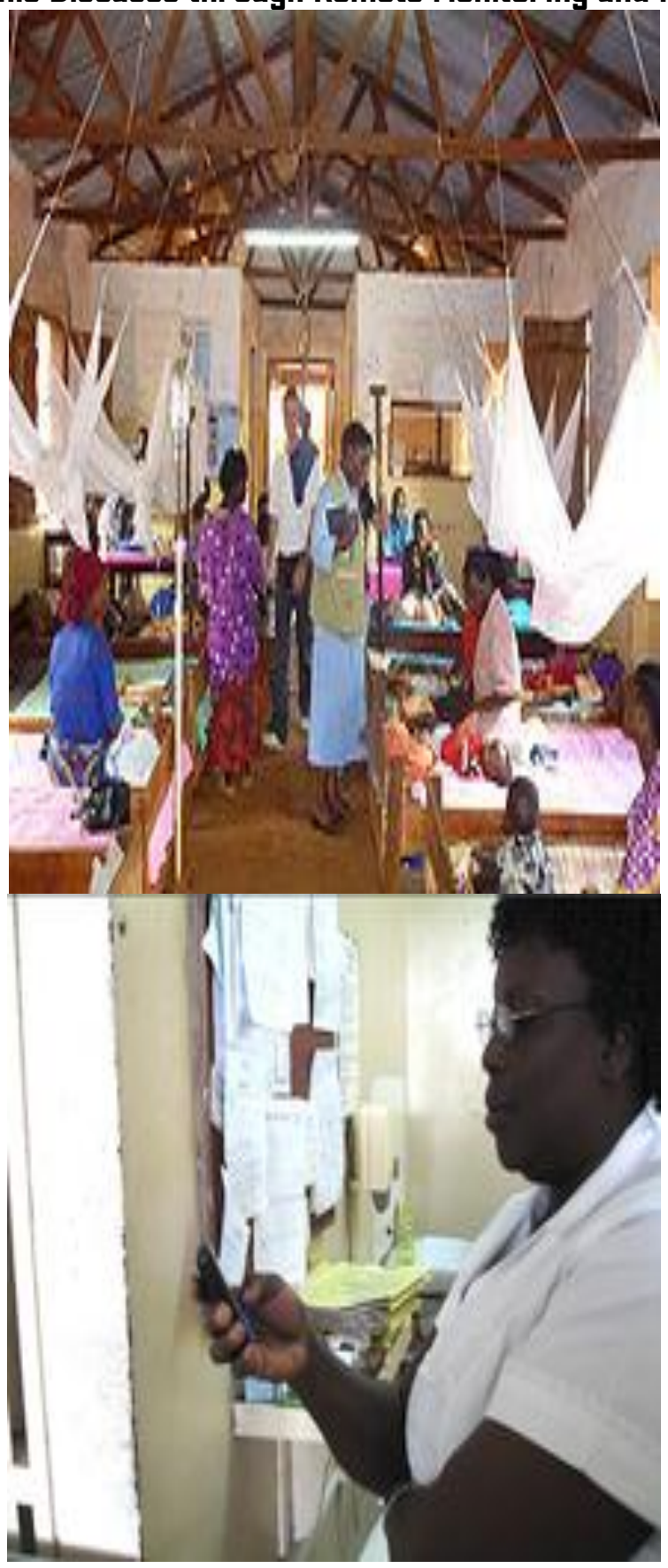


$>$ Chronic disease management represents the greatest health care challenge in many locales. Remote monitoring devices enable patients with serious problems to record their own health measures and send them electronically to physicians or specialists. This keeps them out of doctor's offices for routine care, and thereby helps to reduce health care costs.

$>$ In the area of diabetes, for example, it is crucial that patients monitor their blood glucose levels and gear their insulin intake to proper levels.

$>$ It is possible to use remote monitoring devices at home that record glucose levels instantaneously and electronically send them to the appropriate health care provider. Patients are using "Gluco Phones" that monitor and transmit glucose information to caregivers while also reminding patients when they need to undertake glucose tests.

\section{Helping the Elderly and Expectant Mothers}

$>$ Mobile technology has the potential to help the elderly people to be reminded to take their medicine and communications problems. Patients no longer need to visit doctors' offices to be reminded to take their medicine.

$>$ They can get personal reminders via e-mail, automated phone calls, or text messages.

$>$ Text for Baby is a mobile application for pregnant women. It sends text messages in English and Spanish on how to handle various stages of pregnancy and problems that come up.

$>$ To improve patient awareness, its doctors launched a Mobiles for Health initiative that provides how to take 
care of expectant mothers during pregnancies, early warning signs of possible infant problems, the benefits of family planning, and breast feeding best practices.

\section{Curriculum Integration}

$>$ Integrating mobile technology into nursing curricula is much easier now than it was even 2 years ago.

$>$ Integrating mobile technology into nursing curricula is much easier now than it was even 2 years ago.

$>$ A majority of college students own mobile technology such as a Touch or smart phone, reducing the initial hardware cost.

$>$ Software from leading providers is available for every platform, including tablets, such as the iPad.

$>$ Software references are significantly less expensive than textbooks and if the software is required for coursework, financial aid typically will cover costs.

$>$ The goal of nursing curricula is to educate future nurses to provide safe, patient centered care based on scientific evidence. Mobile technology is an essential 21st century part of achieving that goal.

\section{Patient Safety}

$>$ Nurses use technology resources that "require skills in analysis and synthesis to improve the quality and effectiveness of care." This underscores the need for mobile technology to be fully integrated at all levels of nursing education from associate degree to doctoral practice.

$>$ Mobile software resources help nursing students to be more prepared for clinical assignments and to provide comprehensive nursing care. 
$>$ Using mobile references in the classroom and lab enhances the ability of students to analyze and synthesize critical information and apply it to simulated patient care scenarios.

$>$ Mobile technology allows student nurses to prepare rapidly for patient care at the beginning of each shift within the clinical setting. Nursing faculty report a significant increase in student knowledge and preparation prior to care when they utilize point-of-care resources.

\section{Administration of medication}

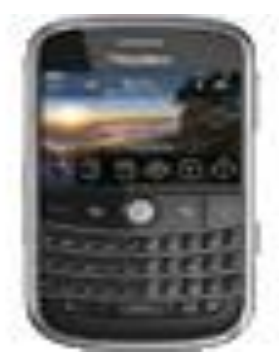

$>$ The most obvious use of mobile technology in nursing education and practice is in preventing medication errors. Nurses are often the final step in the administration process to prevent a medication error.

$>$ Mobile technology provides nursing students and nurses with instant access to safe dose, compatibility, and pharmacokinetic information essential for safe medication administration.

\section{Care Planning}

$>$ Mobile software resources help nursing students to be more prepared for clinical assignments and to provide comprehensive nursing care.

\section{Patient Education}




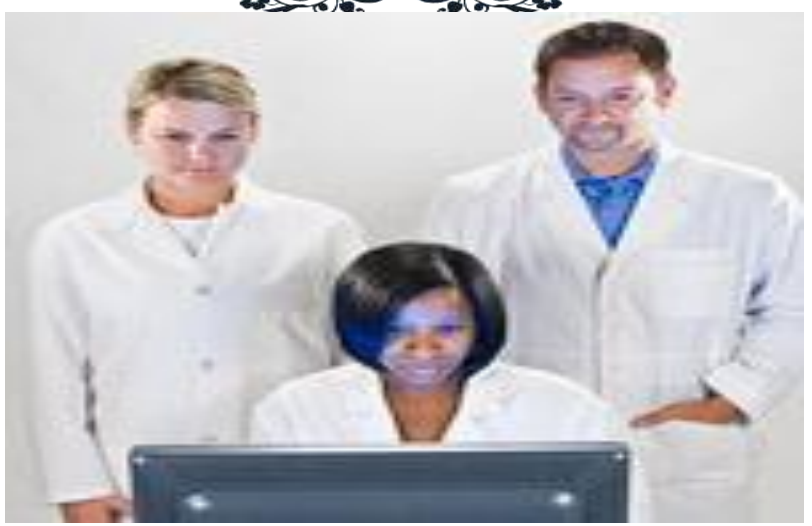

$>$ Resources available via mobile technology enhance the ability of students to provide current evidence-based practice information to their patients in the clinical setting.

$>$ Mobile resources provide step-by-step instructions and photos on hundreds of procedures for students to use prior to or during patient education.

$>$ Patient education is perhaps one of the most challenging aspects of practice for students new to the nursing role. Resources available via mobile technology enhance the ability of students to provide current evidence-based practice information to their patients in the clinical setting.

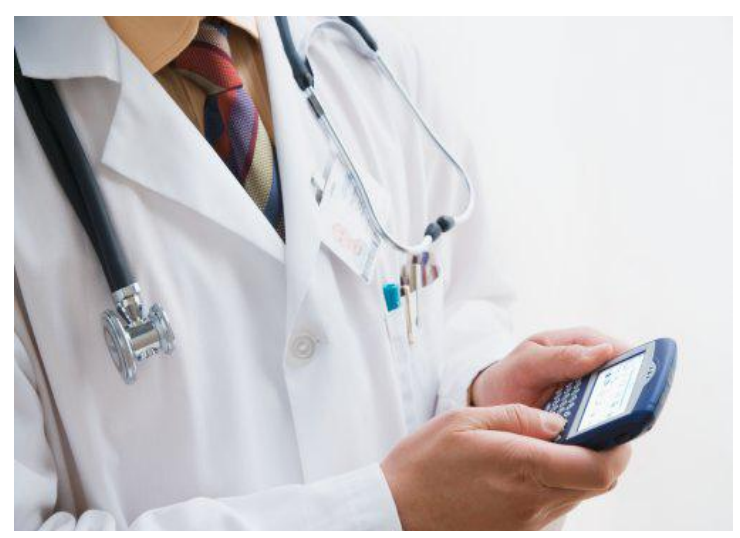

